Kompass

Pneumologie

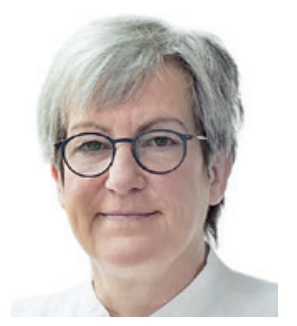

\section{Susanne M. Lang}

Kommissarische Leitung, Klinik für Innere Medizin V, Universitätsklinikum Jena, Jena, Deutschland

Während die pneumologische Onkologie viele Jahre nur für eine kleine Gruppe von Spezialisten verschiedener Disziplinen von Interesse war, ist das Thema durch die rasanten Fortschritte der vergangenen Jahre in den Fokus von Onkologen und Pneumologen gerückt. Neben dem stetig wachsenden Wissen um die Vielzahl von Therapieoptionen, ob allein oder zunehmend auch in Kombination, müssen die Nebenwirkungen der Immuntherapie beachtet werden.

Diese Ausgabe des Kompass Pneumologie greift diesen Trend auf und widmet sich der individualisierten Therapie des Lungenkarzinoms, die die Standardchemotherapie früherer Jahre weitgehend abgelöst hat. Nach der Einführung von Angiogenese-Inhibitoren im Jahr 2007, war der nächste Schritt die Erkenntnis, dass Treibermutationen von einer zielgerichteten Therapie profitieren können. In den letzten Jahren wurden die Kenntnisse über Treibermutationen und Resistenzmechanismen differenzierter und es wurden zahlreiche Tyrosinkinase-Inhibitoren zugelassen. Der Durchbruch kam 2015 mit der Zulassung der Immuntherapie beim nicht kleinzelligen Lungenkarzinom (nonsmall cell lung carcinoma, NSCLC), zunehmend in Kombination mit Chemotherapie. Selbst die Therapie des kleinzelligen Lungenkarzinoms, die lange Zeit keine Fortschritte machte, profitiert von dieser Entwicklung. In den aktuellen Therapiealgorithmen wird die Immuntherapie als Partner der Operation in Studien und die Radiotherapie mit Immunerhaltung im klinischen Alltag

\title{
Lungenkarzinome operativ und medikamentös behandeln: Paradigmenwechsel zur individualisierten Therapie
}

eingesetzt. Erstmals hat auch die zielgerichtete Therapie bei EGFR-mutierten Tumoren in der adjuvanten Situation Vorteile gegenüber der Standardchemotherapie gezeigt. Die Therapieplanung erfordert die Selektion geeigneter Patienten anhand von Biomarkern und Treibermutationen sowie histologischen Kriterien. Die Suche nach verlässlichen Prädiktoren für ein Ansprechen auf Immuntherapie ist jedoch noch nicht abgeschlossen. Lediglich die PD-L1-Expression ist im klinischen Alltag etabliert und im Zulassungstext der PD-1/PD-L1-Antikörper verankert. Die Übersichtsarbeit der Arbeitsgruppe um Giovanni Rossi gibt einen ausführlichen State-of-the-art-Überblick über gängige und neue Biomarker in der Immuntherapie des NSCLCs. Im Fazit vermögen die gegenwärtig bekannten Biomarker beim Lungenkarzinom jedoch nicht zuverlässig zwischen Patienten zu unterscheiden, die auf Immuntherapie ansprechen - oder eben nicht. Limitationen sind u.a. die intra-tumorale Heterogenität und Veränderungen der Biomarker im Verlauf sowie die Schwierigkeiten, ausreichend repräsentatives Material für die Analyse zu gewinnen. Auch die Liquid Biopsy bietet keine perfekte Lösung, da spezifische Histologiebasierte Biomarker, wie Tumor-infiltrierende Lymphozyten (TILs) nicht erfasst werden können. Ob die Mutationsanalyse in der bronchoalveolären Lavage die Diagnostik verbessert, welchen Einfluss Treibermutationen bei Immuncheckpunkt-Inhibition haben und die Rolle von Angiogenese-Hemmern werden in den Wissenstransfers diskutiert.
Das Bündel von Themen wird durch Interventionen beim Kleinzeller und malignen Erguss ergänzt. Die Möglichkeiten der Operation beim kleinzelligen Lungenkarzinom sind individuellen Fällen vorbehalten und sollten in interdisziplinären Tumorboards sorgfältig abgewogen werden. Eine ungewöhnliche pulmonale Nebenwirkung von Tocilizumab wird in einem weiteren Wissenstransfer diskutiert. Das Auftreten schwerer pulmonaler Nebenwirkungen unterstreicht die generelle Wichtigkeit, zwischen Respondern und Non-Respondern auf die Therapie mit Antikörpern zu unterscheiden.

Die Möglichkeit, einen Perikarderguss mittels EUS-B-FNA zu drainieren, zeigt ein ungewöhnlicher Fallbericht.

Zu guter Letzt sollte nicht vergessen werden, dass Patienten mit Lungenkrebs heute länger und mit therapiefreien Phasen überleben. Die Lebensqualität kann durch körperliches Training verbessert werden. Die Trainingssteuerung ist daher ebenso Thema eines Wissenstransfers.

Ich hoffe, die Themen haben Ihr Interesse geweckt, und wünsche Ihnen viel Spaß beim Lesen der neuesten Ausgabe des Kompass Pneumologie.

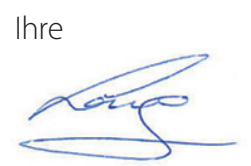

Prof. Dr. Susanne Lang information@karger.com @ (c) 2020 S. Karger GmbH, Freiburg www.karger.com/kkp

Karger ${ }^{\prime \prime}$ -
Prof. Dr. Susanne M. Lang 